\section{Pax6-dependent, but $\beta$-catenin- independent, function of Bcl9 proteins in mouse lens development}

\author{
Claudio Cantù, ${ }^{1}$ Dario Zimmerli, ${ }^{1}$ \\ George Hausmann, ${ }^{1}$ Tomas Valenta, ${ }^{1}$ \\ Andreas Moor, ${ }^{2}$ Michel Aguet, ${ }^{2}$ and Konrad Basler ${ }^{1}$
}

${ }^{1}$ Institute of Molecular Life Sciences, University of Zurich, 8057 Zurich, Switzerland; ${ }^{2}$ Swiss Institute for Experimental Cancer Research, Ecole Polytechnique Fédérale de Lausanne, School of Life Sciences, 1011 Lausanne, Switzerland

$B c 19$ and Bcl91 (Bc19/9I) encode Wnt signaling components that mediate the interaction between $\beta$-catenin and Pygopus (Pygo) via two evolutionarily conserved domains, HD1 and HD2, respectively. We generated mouse strains lacking these domains to probe the $\beta$-catenin-dependent and $\beta$-catenin-independent roles of Bcl9/91 and Pygo during mouse development. While lens development is critically dependent on the presence of the HD1 domain, it is not affected by the lack of the HD2 domain, indicating that $\mathrm{Bc19/91}$ act in this context in a $\beta$-catenin-independent manner. Furthermore, we uncover a new regulatory circuit in which Pax6, the master regulator of eye development, directly activates $B$ c19/91 transcription.

Supplemental material is available for this article.

Received May 26, 2014; revised version accepted August 8, 2014.

Pygopus (Pygo) and Legless (Lgs) were discovered as dedicated Wnt signaling components in Drosophila (Kramps et al. 2002; Parker et al. 2002; Thompson et al. 2002) and Xenopus (Belenkaya et al. 2002). In Drosophila, pygo and $1 g s$ are classified as segment polarity genes, and mutations in their coding sequences lead to a dramatic developmental arrest, reminiscent of wingless mutations (Kramps et al. 2002; Parker et al. 2002; Thompson et al. 2002). They are considered to serve as dedicated and essential $\beta$-catenin transcriptional coactivators. Lgs simultaneously binds Pygo and Arm/ $\beta$-catenin via two evolutionarily conserved homology domains, HD1 and HD2, respectively (Kramps et al. 2002). The prevailing model is that Pan/TCF $>$ Arm $/ \beta$-catenin $>$ Lgs $>$ Pygo serially recruit each other to the DNA in order to efficiently activate Wnt target gene expression, forming a "chain of adaptors." In this model, the sole function of Lgs is to recruit Pygo to $\beta$-catenin (Städeli and Basler 2005).

Vertebrates feature two pygo genes, PYGO1 and PYGO2, and two lgs homologs, BCL9 and BCL91 (BCL9/91). In contrast to $\beta$-catenin loss-of-function mutants, Pygo1/Pygo2

[Keywords: Wnt; mouse; Bcl9; Pygopus; lens induction; Pax6] Corresponding author: basler@imls.uzh.ch

Article is online at http://www.genesdev.org/cgi/doi/10.1101/gad.246140.114. double-knockout mice proceed normally throughout gastrulation and die later during embryonic development from a series of tissue-specific defects. Pygo1 knockout mice are viable and fertile, with no apparent phenotype. Compound Pygo1/Pygo2 knockout mutants are indistinguishable from Pygo2 knockout, suggesting that Pygo2 plays the more important role during development ( $\mathrm{Li}$ et al. 2007; Schwab et al. 2007; M Aguet, unpubl.). Unlike in Drosophila, in mice, Pygol and Pygo2 are now considered to be tissue-specific Wnt pathway components.

$\mathrm{Bcl}$ /91 are necessary for the expression of Wnt targets in normal intestinal epithelia (Deka et al. 2010) and the myogenic progenitors during muscle regeneration, where a reduction of $\mathrm{Bcl} / 91$ inhibits the Wnt-driven myogenic differentiation (Brack et al. 2009): This indicates that Bcl9/91 are required for the expression of key subsets of $\beta$-catenin target genes. Consistent with this, mutating $\beta$-catenin such that it cannot bind $\mathrm{Bc1} 9 / 91$ ( $\beta$-catenin-D164A) results in embryonic lethality at mid-gestation, around embryonic day 10.5 (Valenta et al. 2011). In addition, a growing body of evidence indicates that $\mathrm{Bcl} / 91$ and their interaction with $\beta$-catenin are important not only for normal cell functions but also in different types of tumors (Mani et al. 2009; Deka et al. 2010; Brembeck et al. 2011; Takada et al. 2012). When their interaction is abrogated in these tumors, an attenuated migratory potential is observed, accompanied by a decrease in the expression of the Lgr $5^{+}$-associated stem cell genes (de la Roche et al. 2012; Kawamoto et al. 2012; A Moor, P Anderle, C Cantù, N Wiedemann, F Baruthio, P Rodriguez, J Deka, S André, T Valenta, B Györffy, et al., in prep.). The conclusion from these studies was that, in mammals, Bcl9/91 retains a critical global function for $\beta$-catenin-mediated target gene control, at least in a tissue-specific manner. Based on comparison with the Pygo2 knockout situation, this role can be considered, at least in part, Pygo-independent. However, the molecular function of Bcl9/91 during mammalian development and tissue homeostasis remains largely unexplored.

Here we present an analysis of a series of $B c 19 / 91$ knockin mouse strains in which, via the deletion of the HD1 or HD2 domains, Bcl9/9l lose the ability to bind Pygo or $\beta$-catenin, respectively. We found that both interactions are relevant for development, and their individual disruption leads to embryonic lethality. Unexpectedly, however, we found that Bc19/91 contribute in a Pygo-dependent, but $\beta$-catenin-independent, fashion to eye lens formation, revealing for the first time that Bcl9/91 have functions that are separate from canonical Wnt signaling. Moreover, we provide molecular evidence for a novel genetic circuit containing Bcl9/91 and Pygo that does not involve $\beta$-catenin: Pax6, the master regulator of eye differentiation, directly activates $B c 19$ and $B c 191$ transcription. Pygo2 is required for the function of Bc19/91, and the Bcl9/91-Pygo2 complex appears to sustain Pax6 expression. This reveals that, independently of canonical Wnt signaling, Bcl9/91 and Pygo2 constitute a critical molecular unit that responds to tissue-specific regulators, such as, in the lens, the Pax6 transcription factor.

(C) 2014 Cantù et al. This article is distributed exclusively by Cold Spring Harbor Laboratory Press for the first six months after the full-issue publication date (see http://genesdev.cshlp.org/site/misc/terms.xhtml). After six months, it is available under a Creative Commons License (Attribution-NonCommercial 4.0 International), as described at http:// creativecommons.org/licenses/by-nc/4.0/. 


\section{Results and Discussion}

Pygo2 is assumed to be recruited by Bcl9/91 to promote the output of the Wnt signaling pathway. However, clear evidence that the Bc19/91-Pygo2 interaction is relevant during mammalian development is lacking. To specifically investigate this, we generated constitutive knock-in $B c 19$ and Bcl91 alleles that carry an in-frame deletion of the conserved HD1 (Fig. 1A; Supplemental Fig. S1), the domain responsible for binding to Pygo proteins (Kramps et al. 2002; Städeli and Basler 2005; Fiedler et al. 2008). This deletion abrogates the recruitment of Pygo proteins to the $\beta$-catenin transcriptional complex (Fig. 1A; Mosimann et al. 2009). Indeed, via GST pull-down assays, we confirmed that a $\mathrm{Bcl} 9$ protein lacking the $\mathrm{HD} 1$, when incubated with total protein extracts obtained from 12.5-d postcoitum (dpc) embryos, fails to bind Pygo2 but still binds $\beta$-catenin (Fig. 1B), supporting the validity of the rationale and indicating that a deletion of this domain does not lead to improper folding of the protein. Pygol was not detectable in our experiments, consistent with the previous finding
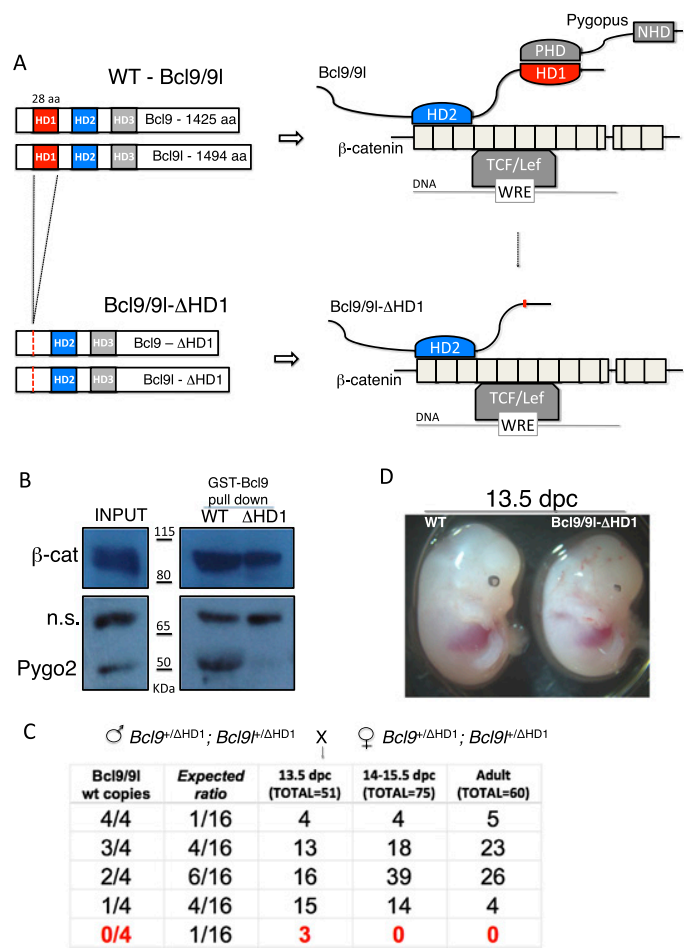

Figure 1. The Bcl9/91-Pygo2 interaction is necessary during mouse embryonic development. (A) Wild-type and $\Delta \mathrm{HD} 1$ mutant $\mathrm{Bcl} 9$ and Bcl9l proteins; at the right is presented the variation in the "chain of adaptors" induced by the deletion of the HD1 domain. (WRE) Wntresponsive element; (PHD) plant homeodomain; (NHD) N-terminal homology domain. (B) A GST-Bcl9- $\Delta \mathrm{HD}$ protein, when incubated with protein extracts obtained from 12.5-dpc wild-type embryos, loses the ability to interact with Pygo2 but maintains the ability to pull down $\beta$-catenin; nonspecific signal (n.s.), obtained with Pygo2 antibody, was used as a loading control. $(C)$ Crossings between doubleheterozygous mice: All of the possible genetic combinations are grouped based on the number of wild-type alleles of Bcl9/9l, from four out of four to zero out of four. Double-homozygous $\Delta \mathrm{HD} 1$ mutant mice (Bc19/91- $\Delta \mathrm{HD} 1)$ are never found after $13.5 \mathrm{dpc}$, indicating embryonic lethality. (D) Bcl9/9l- $\Delta \mathrm{HD} 1$ mice at $13.5 \mathrm{dpc}$ appear slightly smaller but do not display any obvious developmental defect, apart from an eye malformation (see Fig. 2), when compared with control littermates (wild type [WT]). that it is only weakly expressed later during embryonic development (Li et al. 2004). For this reason, in our study, we only considered the contribution of Pygo2.

Double-heterozygous knock-in mice $\left(B c 19^{\Delta \mathrm{HD} 1 /+}\right.$; $B c 191^{\mathrm{HD} 1 /+}$ ) develop normally and do not display any obvious defect. Interestingly, mice homozygous mutant only in either $B c 19$ or Bcl91 (that is, $B c 19^{\Delta \mathrm{HD} 1 / \Delta \mathrm{HD} 1}$; $\mathrm{BcllO}^{+/+}$and $\mathrm{Bcl} \mathrm{9}^{+/+} ; \mathrm{Bcl} 19 \mathrm{I}^{\mathrm{\Delta HD1} / \Delta \mathrm{HD} 1}$ ) are born in $\sim 50 \%$ of the expected Mendelian ratios. This indicates a substantial redundancy between $\mathrm{Bcl} 9$ and $\mathrm{Bcl} 91$, at least concerning the functions mediated by the HD1. In contrast, no doublehomozygous mutants $\left(B c 19^{\Delta \mathrm{HD} 1 / \Delta \mathrm{HD} 1} ; B c 191^{\Delta \mathrm{HD} 1 / \Delta \mathrm{HD} 1}\right)$ were ever found later than $13.5 \mathrm{dpc}$ (Fig. 1D), indicating embryonic lethality at this stage. Throughout the study, to simplify the analysis and strengthen the validity of the conclusion drawn, we investigate the effects of the simultaneous deletion in both genes and refer to these mice as Bc19/91- $\Delta$ HD1.

Of note, the complete loss of Bcl9/91 (Bc19/91 knockout) causes an earlier lethality, occurring between 9.5 and 10.5 dpc (Supplemental Fig. S2; M Aguet, unpubl.); this suggests the interesting notion that, between 9.5 and $13.5 \mathrm{dpc}, \mathrm{Bcl} / 91$ have Pygo-independent functions. Bc19/91- $\Delta$ HD1 mutant embryos at $13.5 \mathrm{dpc}$ are slightly smaller than the control embryos (Fig. 1C); the cause of the lethality is currently under investigation. Remarkably, the timing of the embryonic lethality of $B c 19 / 91-\Delta \mathrm{HD} 1$ mice is similar to that of Pygo1/Pygo2 knockout embryos (Supplemental Fig. S3). The time point differs slightly from that previously reported (Li et al. 2007; Schwab et al. 2007). This minor difference may be due to an influence of the genetic background or a difference between the independently generated genomic manipulations. These results indicate that an important aspect of $\mathrm{Bcl}$ /9/91 function is mediated by the HD1 domain. With the caveat that other, so far unrecognized proteins may also interact with $\mathrm{Bcl} / 9 / 91$ via the HD1 domain, the results show that the Pygo2-Bc19/91 interaction is required for mouse development as of 13.5 dpc.

An aspect in which the $B c 19 / 91-\Delta H D 1$ embryos are identical to the Pygo2 knockout ones is that they display an obvious eye defect: The lens is absent (Fig. 2). Of note, the developing eye is one of the head structures displaying a marked expression of both $\mathrm{Bcl} 9$ and $\mathrm{Bcl} 191$ (Supplemental Fig. S4). The lens defect also often appears in triple-mutant mice, which retain only one wild-type allele of $B c 19$ or $B c 191$ (that is $B c 19^{+/ \Delta \mathrm{HD} 1} ; B c 191^{\Delta \mathrm{HD} 1 / \Delta \mathrm{HD} 1}$ and $B c 19^{\Delta \mathrm{HD} 1 / \Delta \mathrm{HD} 1}$; $\left.B c 191^{+/ \Delta \mathrm{HD} 1}\right)$. Interestingly, such genetic combinations most often lead only to a unilateral lens loss, a further indication that $\mathrm{Bcl} 9$ and $\mathrm{Bcl} 91$ are highly redundant with respect to the functions mediated by Pygo 2 binding.

During eye development, the surface head ectoderm thickens to form the lens placode and at $10.5 \mathrm{dpc}$ invaginates to generate the lens pit (Lang 2004). In Bc19/91- $\Delta \mathrm{HD} 1$ embryos, lens development is blocked at this stage: The surface head ectoderm does not form the eye placode, the first morphological sign of lens development (Fig. 2, bottom panels), indicating that the Bc19/91-Pygo2 complex is required early at the onset of lens formation. Also, in Bc19/91 knockout embryos, lens development is arrested at this stage (Supplemental Fig. S2E,F).

Interestingly, a positive role of Bcl9/91 in lens formation is in contradiction with the notion that Wnt signaling must be turned off during lens development (Smith et al. 2005; Machon et al. 2010). Therefore, we reasoned 


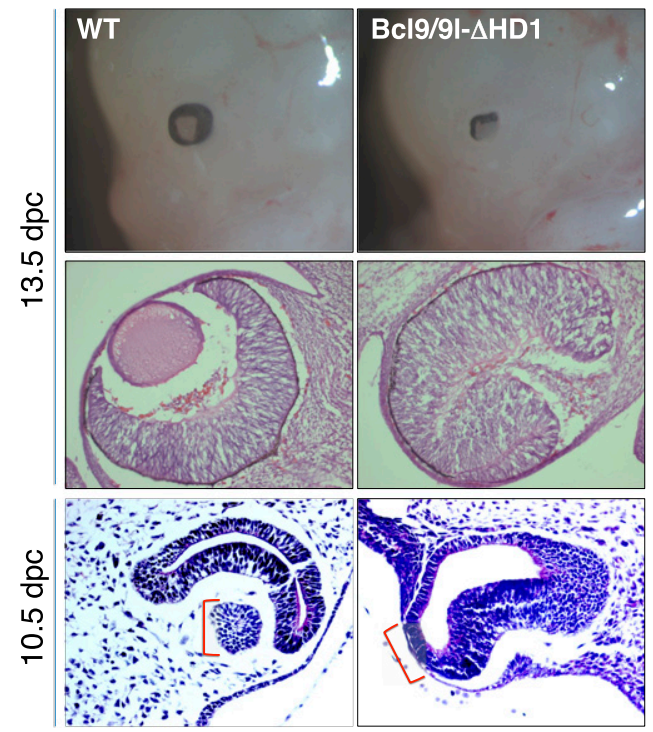

Figure 2. Bcl9/91 has a role in early lens development. (Top panels) Bc19/91- $\Delta$ HD1 double-mutant embryos display an eye defect highly reminiscent of the one previously described for Pygo2 loss of function (Song et al. 2007). (Middle panels) The dissection of the eye structure at this stage (i.e., $13.5 \mathrm{dpc}$ ) shows a complete absence of the lens accompanied by an enlarged developing retina, a feature that resembles the lens-specific conditional loss of Pax6 (AsheryPadan 2000). (Bottom panels) Bc19/91- $\Delta \mathrm{HD} 1$ mutant embryos at 10.5 $\mathrm{dpc}$ fail to induce eye placode thickening and subsequent lens pit formation. A square bracket indicates the lens vesicle in the wildtype (WT) eye and the region of the surface head ectoderm that, in Bc19/91- $\Delta$ HD1 mutants, despite lying close to the optic vesicle, fails to form a lens placode.

that Bc19/91 might not act as Wnt signaling components in this tissue. This hypothesis predicts that, if $\mathrm{Bcl} / 91 \mathrm{can}$ still bind Pygo 2 but not $\beta$-catenin, the lens will develop correctly. To assess this possibility, we created a knock-in mouse strain carrying an in-frame deletion of HD2 $(\Delta \mathrm{HD} 2)$ in Bcl9 and Bcl91 (Fig. 3A; Supplemental Fig. $\mathrm{S} 5)$ : We and others have previously shown that, in the absence of the HD2 domain, Bcl9/91 can still bind to Pygo2 but not to $\beta$-catenin (Fig. 3B; Kramps et al. 2002; Städeli and Basler 2005).

Of note, double-mutant embryos $\left(B c 19^{\Delta \mathrm{HD} 2 / \Delta \mathrm{HD} 2}\right.$; $B c 191^{\Delta \mathrm{HD} 2 / \Delta \mathrm{HD} 2}$, referred to as $\left.B c 19 / 91-\Delta \mathrm{HD} 2\right)$, die at $\sim 10.5 \mathrm{dpc}$, earlier than Bc19/91- $\Delta$ HD1 mutants (Fig. 3C). This is consistent with the lethality caused by the $\beta$-catenin-D164A mutation, which also abrogates the Bc19/91- $\beta$-catenin interaction (Valenta et al. 2011). A corollary of this is that early $\mathrm{Bcl} / 91$ functions are independent of Pygo 2 but are mediated by $\beta$-catenin; in other words, a truncated "chain of adaptors" lacking Pygo2 can sustain mouse development until at least embryonic day 13.5 .

In order to investigate whether the loss of the Bcl9/91$\beta$-catenin interaction also blocks lens formation at 10.5 dpc, we compared eye development in Bc19/91 wild-type, $B c 19 / 91-\Delta \mathrm{HD} 1$, and Bc19/91- $\Delta \mathrm{HD} 2$ embryos (Fig. 3E). In wild-type embryos at $10.5 \mathrm{dpc}$, the lens pit is clearly distinguishable. In $B c 19 / 91-\Delta \mathrm{HD} 1$ mutants, the lens pit is not forming. Importantly, in Bc19/91- $\Delta \mathrm{HD} 2$ embryos, the lens pit is developing normally (Fig. 3E, right panel). This result indicates that, during lens formation, the Bcl9/91-
Pygo2 complex functions independently of the physical interaction with $\beta$-catenin.

In addition, we incorporated the lens-cre transgene, a widely used lens-specific Cre recombinase driver (Ashery-

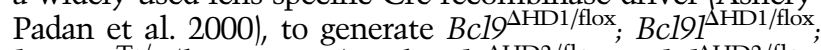
lens-cre $e^{\mathrm{Tg} /+}($ lens- $\Delta \mathrm{HD} 1)$ and $B c 19^{\Delta \mathrm{HD} 2 / \text { flox }} ; B c 191^{\Delta \mathrm{HD} 2 / \text { flox; }}$ lens-cre ${ }^{\mathrm{Tg} /+}$ (lens- $\left.\Delta \mathrm{HD} 2\right)$ mice. Compared with control animals, at birth, lens- $\Delta$ HD1 mice have smaller lenses. The same effect is obtained upon the complete loss of

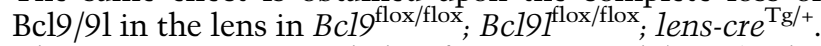
This is in agreement with data from Song et al. (2007), who found that lens-cre-driven Pygo2 deletion does not entirely recapitulate the lens loss induced by constitutive deletion of Pygo2. Importantly, however, lens- $\Delta \mathrm{HD} 2$ pups have normally shaped lenses and are in all cases indistinguishable from control littermates (Supplemental Fig. S6). This indicates that the Bc19/91-Pygo2 complex does not require $\beta$-catenin to mediate its role during lens development.

To our knowledge, this is the first report demonstrating that Bcl9/91 act outside the canonical Wnt signaling cascade. A growing body of evidence indicates that, in

A

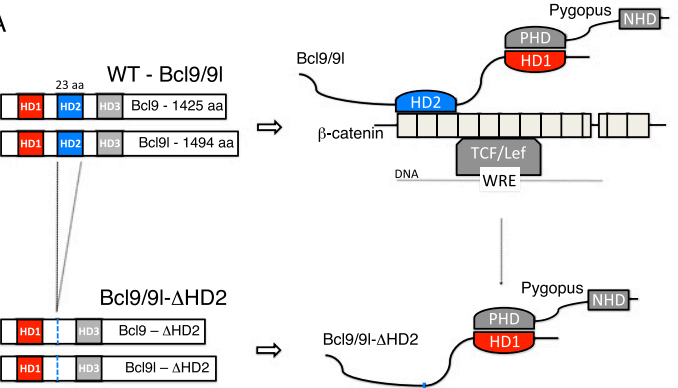

B
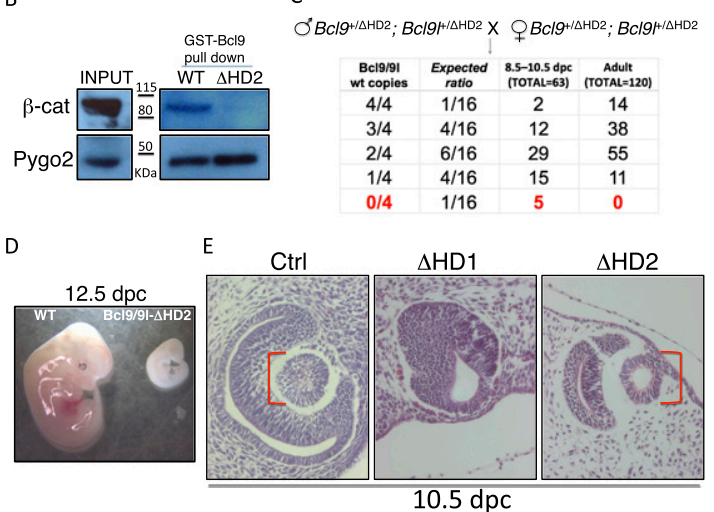

Figure 3. Bcl9/91 act independently from $\beta$-catenin during lens development. (A) Wild-type (WT) and $\Delta \mathrm{HD} 2$ mutant Bcl9 and Bcl91 proteins; at the right is presented the variation in the "chain of adaptors" induced by deleting the HD2 domain: The interaction between $\mathrm{Bcl} / 91$ and $\beta$-catenin is abrogated, and the complex Bcl9/ 91-Pygo2 can act independently from canonical Wnt signaling. $(B) \mathrm{A}$ GST-Bcl9- $\Delta \mathrm{HD} 2$ protein loses the ability to interact with $\beta$-catenin but maintains the ability to pull down Pygo2. $(C)$ No doublehomozygous $\triangle \mathrm{HD} 2$ mutant mice are ever scored after birth, suggesting embryonic lethality. $(D)$ The few embryos found at $12.5 \mathrm{dpc}$ displayed an evident developmental block at 10.5 dpc. $(E)$ At 10.5 dpc, wild-type surface head ectoderm invaginates to form the lens pit; in Bc19/91- $\Delta \mathrm{HD} 1$ embryos, this process is arrested (cf. the middle and left panels). At the same stage, Bcl9/91- $\Delta \mathrm{HD} 2$ embryos have a correctly shaped lens pit, demonstrating that, in this process, Bcl9/ 91 function is independent from $\beta$-catenin. 
mice, Pygo2 has tissue-specific, Wnt-independent functions in the testis (Nair et al. 2008; Cantù et al. 2013) and lens (Song et al. 2007). Also, in Drosophila, Pygo was recently reported to act in a $\beta$-cateninindependent manner during heart development (Tang et al. 2013, 2014). It will be critical to establish whether other Wnt-independent functions of Pygo2 require the interaction with $\mathrm{Bcl} / 9 / 91$ and whether these functions are conserved among species.

Our finding that the Bcl9/91-Pygo 2 complex functions independently of $\beta$-catenin in eye development implies that, in this tissue, these factors also act independently from upstream canonical Wnt signals. The abrogation of the Bc19/91-Pygo2 interaction causes a lens arrest very reminiscent of Pax6 loss in this tissue (AsheryPadan et al. 2000). Therefore, we reasoned that Pax6 might lie upstream of Bcl9/91 function. To test whether Pax6 controls Bc19, Bcl91, and Pygo2 expression, we exploited the mouse-derived lens cell line $\alpha$ TN4, a widely used model for lens cell differentiation. This cell line expresses Pax6 and its direct transcriptional target, $\alpha \mathrm{A}$-crystallin, encoded by the Cryaa gene (Yang and Cvekl 2005); importantly, Bc19/91 and Pygo2 are also expressed (Fig. 4A), making this cell line a suitable model for studying their regulation.

We identified in silico Pax6 consensus sequences between $-5 \mathrm{~kb}$ and $+1 \mathrm{~kb}$ of the transcriptional start site (TSS) of Bc19, Bc191, and Pygo2. To determine whether any of these serve as bona fide Pax6-binding sites, we performed chromatin immunoprecipitation (ChIP) experiments. We designed PCR primers to amplify the Pax6-binding sites located within evolutionarily conserved regions (Supplemental Fig. S7). Indicative of Pax6 binding, we found that specific regions upstream of $B c 19$ and $B c 191$ are enriched in our ChIP assay when using an anti-Pax6 antibody (Fig. 4B). Note that the Cryaa promoter, our positive control, displays a comparable enrichment, while no enrichment is observed in several other genomic regions (e.g., Prm2 promoter) (Fig. 4B). To probe the relevance of Pax6 binding within these regions, we treated $\alpha \mathrm{TN} 4$ cells with siRNA against Pax6 (siPax6): Pax6 mRNA levels were reduced to $<5 \%$ of normal expression, causing a down-regulation of its target $\alpha$ A-crystallin (Fig. 4C). Of note, Pax6 depletion also resulted in the down-regulation of both $\mathrm{Bcl} 9$ and Bcl91 transcripts.

To test whether Pax6 also affected Bcl9/91 expression in vivo, we monitored Bc19/91 expression in the developing eye in the absence of Pax6 function. To this end, we exploited Small eye (Sey) mice that bear a Pax6-truncating mutation, a good model to investigate Pax6 loss of function in vivo (Hogan et al. 1986; Hill et al. 1991). Homozygous Pax6 $6^{\text {Sey/Sey }}$ embryos can be distinguished as early as $10.5 \mathrm{dpc}$ by the phenotype that, at this stage, the ectoderm does not invaginate to form a lens pit (Hogan et al. 1986). In wild-type mice, we found Bcl9l (Fig. 4D) and Bcl9 (Supplemental Fig. S4) proteins to be highly expressed in the invaginating surface head ectoderm, overlapping with the Pax6 expression domain

C

E

F

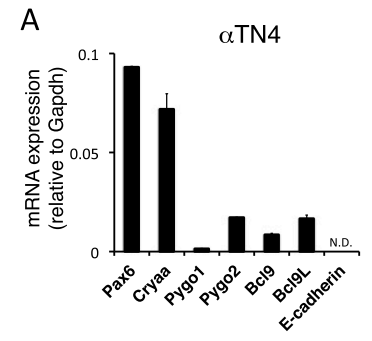

B ChIP $-\alpha P a x 6 / \lg G$
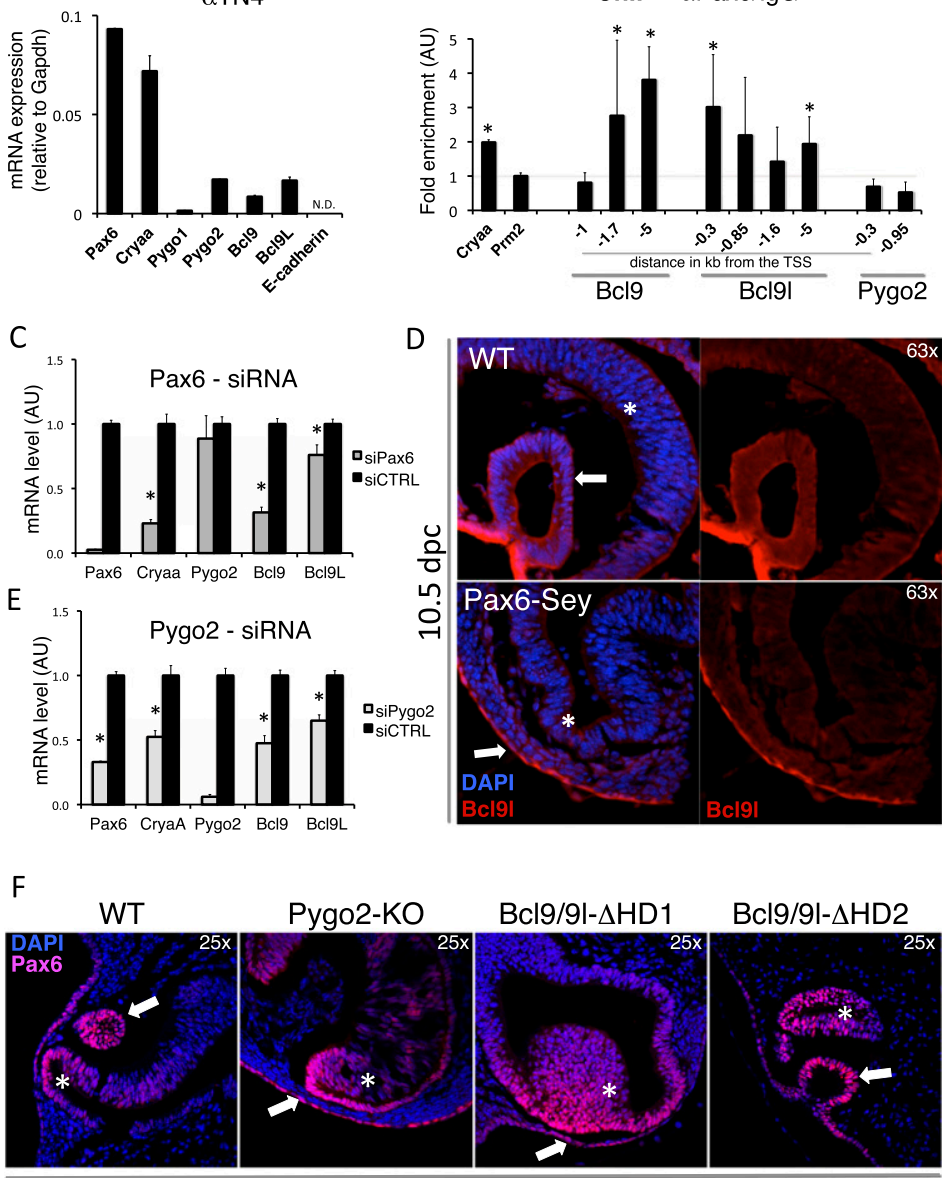

$10.5 \mathrm{dpc}$

Figure 4. $B c 19$ and $B c 191$ are direct transcriptional targets of Pax6. (A) The mousederived lens cell line $\alpha$ TN4 expresses Bc19, Bc191, and Pygo2 together with lensspecific genes such as Pax6 and Cryaa. (B) ChIP performed on the chromatin extracted from $\alpha$ TN4: Specific regions upstream of Bcl9 and Bcl9l are enriched when the chromatin is immunoprecipitated with an anti-Pax6 antibody, indicative of Pax6-binding events. No such enrichment was scored within the Pygo2 promoter. Cryaa and Prm2 promoters constitute the positive and the negative controls, respectively. The enrichment is expressed as a ratio between anti-Pax6 and control IgG immunoprecipitation reactions. (TTS) Transcriptional start site. $(C)$ When $\alpha \mathrm{TN} 4$ is treated with siRNA against Pax6, Pax6 mRNA is reduced to $<5 \%$ of the control. As its direct target, Cryaa, Bcl9 and Bc19l levels are also reduced upon Pax6 depletion. Of note, Pygo2 transcription is not altered. $(D) \mathrm{A}$ loss-of-function mutation of Pax6 in vivo leads to a diminished Bcl9l expression in the surface head ectoderm at the onset of lens pit formation. Arrows mark the surface head ectoderm (already forming the lens pit in the wild-type [WT] embryo); the asterisks indicate the diencephalic protrusion that constitutes the presumptive retina. DAPI is in blue and marks cell nuclei; Bcl9l is in red. $(E)$ If $\alpha$ TN4 cells are treated with siRNA against Pygo2, Pax6 is down-regulated. In such experiments, Bcl9 and Bcl91, together with Cryaa, are also affected, very likely due to a secondary effect of Pax6 down-regulation. (AU) Arbitrary units. (F, left panel) At $10.5 \mathrm{dpc}$, the surface head ectoderm that forms the lens pit expresses high levels of Pax6; at this stage in wild-type embryos, Pax6 appears to be stronger in the surface ectoderm (white arrows) than in the presumptive retina (asterisks). In Pygo2 knockout (Pygo2-KO) and $B c 19 / 91-\Delta \mathrm{HD} 1$ embryos, this ratio is inverted. Please note that to detect the scant Pax6 within the surface head ectoderm, the signal must be enhanced; this explains the apparent brighter signal in mutant retinas. In $B c 19 / 91$ $\Delta \mathrm{HD} 2$ embryos, the developing lens pit displays a Pax6 expression comparable with the wild type. Statistically significant values are evidenced by asterisks, which indicate a $P$-value $<0.05$ calculated using an unpaired one-tail $t$-test.

(Callaerts et al. 1997). In $\mathrm{Pax}^{\text {Sey/Sey }}$ embryos, the ectodermal Bc191 expression appears to be strongly reduced compared with wild-type controls (Fig. 4D, cf. top and 
bottom panels). The same is true for Bcl9 (data not shown). Taken together, our in vitro and in vivo results strongly suggest that Pax6 is required for Bcl9 and Bcl9l transcription in developing lens cells, making them downstream effectors in this cascade. Of note, $B c 191$ was also reported as a putative Pax6 target based on ChIP experiments from lenses of newborn mice combined with high-density oligonucleotide array hybridizations (ChIP-chip) (Xie et al. 2013); this is an independent validation of our findings.

In Pygo2-null mice, Pax6 levels are reduced in the surface head ectoderm at $10.5 \mathrm{dpc}$; on the other hand, when Pax6 function is lacking, Pygo2 expression is unaffected (Fig. 4C; Song et al. 2007, N Vilain and M Aguet, unpubl.). Therefore, Pygo2 appears to be genetically upstream of Pax6. We observed that abrogating the interaction between Bc19/91 and Pygo2 leads to a very similar decrease of Pax6 expression in the surface head ectoderm at $10.5 \mathrm{dpc}$ (Fig. 4F), indicating that Bc19/91 not only are Pax6 targets but also act together with Pygo2 upstream of Pax6. The effect on Pax6 expression is not observed in the Bc19/91- $\Delta$ HD2 developing lens, lending further support to the notion that, in this context, Bc19/91 act in a $\beta$-catenin-independent fashion. The epistatic hierarchy between Bc19/91-Pygo2, Pax6, and Bc19/91 raises the prediction that, if Pygo2 expression was altered in lens cells, Pax6 and Bc19/91 expression would be affected accordingly. To test this, we treated $\alpha \mathrm{TN} 4$ cells with siRNA targeting Pygo2 (siPygo2): Consistently, Pygo2 down-regulation causes a decrease in Pax6 mRNA level and diminished levels of its target genes, Cryaa, Bcl9, and Bcl9l (Fig. 4E). Taken together, our results reveal the existence of a novel genetic regulatory circuit in which Bc19/91-Pygo2 act upstream of Pax6, which in turn activates Bc19 and Bcl91. Thus, in a $\beta$-catenin-independent manner, a Bcl9/91-Pygo2 complex sustains correct lens formation during mouse development (Fig. 5).

$\mathrm{Bcl} 9$ and Bc19l, in a manner requiring Pygo2 binding, thus emerge as new downstream effectors of Pax6 during lens development. In this context, their action does not appear to be modulated by upstream canonical Wnt signals but by a tissue-specific transcription factor, Pax6, the master regulator of the lens-specific differentiation program. The molecular mechanism by which the Bcl9/ 91-Pygo2 complex achieves its tissue-specific function in lens differentiation is an interesting line of future investigation. One possibility is that the main function of the Bc19/91-Pygo2 complex is to sustain Pax6 expression. Consequently, by activating Bc19/91, Pax6 would buttress its own expression, creating a positive feedback loop, a very common mechanism in biological systems, instrumental to produce an all-or-none response to a signal (Brandman et al. 2005).

It is currently widely accepted that, in the lens, the Wnt pathway must be turned off (Smith et al. 2005), and a recent study discovered that this inhibition is mediated at least in part by Pax6 (Machon et al. 2010). It is therefore tantalizing to speculate that $\mathrm{Bcl} / 91$ and Pygo2 not only act independently from $\beta$-catenin but, together with Pax6, are involved in the inhibition of the Wnt-induced genetic program, thus ensuring that lens development proceeds correctly. To test this speculation, a more detailed understanding of the timing and nature of Wnt signaling and its targets in the developing lens is needed. Our results provide a novel circuit and a novel paradigm (a $\beta$-catenin-independent Bcl9/91-Pygo2 module) that need to be integrated into the emergent picture.

\section{Materials and methods}

\section{Generation of Bc19/91 knock-in mouse strain}

Knock-in mutants in $\mathrm{Bcl}$ and $\mathrm{Bcl} 191$ were generated by standard techniques (inGenious Targeting Laboratory). Briefly, the targeting vector was electroporated into BA1 (C57BL/6 × 129/SvEv) hybrid embryonic stem cells. After selection with the antibiotic G418, surviving clones were expanded for PCR and Southern blotting analyses to confirm recombinant embryonic stem cell clones. Mouse embryonic stem cells harboring the knock-in allele were microinjected into C57BL/6 blastocysts. Resulting chimeras were bred to wild-type C57BL/6N mice to generate F1 heterozygous offspring. Neo cassette excision was obtained by crossing heterozygous knock-in animals with mice expressing Flp recombinase. All mouse experiments were performed in accordance with Swiss guidelines and approved by the Veterinarian Office of the Kanton of Zurich, Switzerland.

\section{Cell culture and quantitative RT-PCR}

The $\alpha$ TN4 cell line was cultured in DMEM supplemented with $10 \%$ FBS at $37^{\circ} \mathrm{C}$. Total RNA was extracted using TRIzol (Invitrogen). Quantitative real-time SYBR Green-based PCR reactions were performed in triplicate and monitored with the ABI Prism 7900HT system (Applied Biosystem). All primers are listed in the Supplemental Material.

\section{ChIP}

$\alpha \mathrm{TN} 4$ were fixed with $1 \%$ formaldehyde for $10 \mathrm{~min}$ at room temperature and lysed in a nuclei lysis buffer $(1 \%$ SDS, $50 \mathrm{mM}$ Tris, $10 \mathrm{mM}$ EDTA, protease inhibitors). Chromatin was sonicated to a size between 200 and 1000 base pairs using a Covaris focused ultrasonicator (10 times for $1 \mathrm{~min}$, $10 \%$ duty cycle, $5 \%$ intensity, 100 cycles per burst). The antibodies and the primers used are listed in the Supplemental Material.

\section{Immunofluorescence}

Previously fixed cryosections were blocked with $10 \%$ goat serum and $0.3 \%$ Triton-X-100 in PBS and incubated overnight at $4{ }^{\circ} \mathrm{C}$ with the following antibodies: $\alpha$-Pax6 (Millipore), $\alpha$-Bcl9 (Abcam), $\alpha$-Bc191 (Abnova), and $\alpha$ - $\beta$-catenin (Cl. 14, BD Transduction Laboratories). Slides were then incubated with a fluorescently labeled secondary antibody (Alexa 488 goat anti-mouse or Alexa 555 goat anti-rabbit; 1:500). Nuclei were stained with DAPI (1:1000; Sigma).

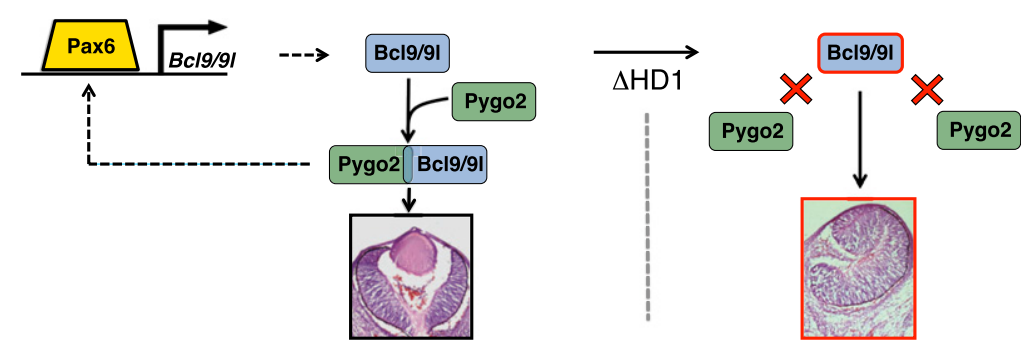

Figure 5. Our data indicate that Pax6 is required for the transcription of $B c 19 / 91$. Bc19/91 then assemble with Pygo2 to ensure a correct lens development; the lens development is arrested when the Bcl9/91-Pygo2 interaction is abolished. The complex Bc19/91-Pygo2 also lies genetically upstream of Pax6, possibly with the function of sustaining its expression. 


\section{Acknowledgments}

We are much indebted to Gabriel Cavalheiro and Fabienne Murphy-Seiler for experimental help; Qing Xie, Christian Mosimann, and Bahar Degirmenci for precious inputs and discussion; Lukas Sommer, Peter Gruss, Christian Grimm, Richard Lang, Anastassia Stoykova, and Ales Cvekl for sharing reagents and mouse strains; and Martin Moser, Eliane Escher, and Charlotte Burger for technical support. This work was supported by the Swiss National Science Foundation (SNF) and grants from the Forschungskredit of the University of Zurich (to C.C.).

\section{References}

Ashery-Padan R, Marquardt T, Zhou X, Gruss P. 2000. Pax6 activity in the lens primordium is required for lens formation and for correct placement of a single retina in the eye. Genes Dev 14: 2701-2711.

Belenkaya TY, Han C, Standley HI, Lin X, Houston DW, Heasman J, Lin X. 2002. pygopus encodes a nuclear protein essential for wingless/Wnt signaling. Development 129: 4089-4101.

Brack AS, Murphy-Seiler F, Hanifi J, Deka J, Eyckerman S, Keller C, Aguet M, Rando TA. 2009. BCL9 is an essential component of canonical Wnt signaling that mediates the differentiation of myogenic progenitors during muscle regeneration. Dev Biol 335: 93-105.

Brandman O, Ferrell JE, Li R, Meyer T. 2005. Interlinked fast and slow positive feedback loops drive reliable cell decisions. Science 310: 496498.

Brembeck FH, Wiese M, Zatula N, Grigoryan T, Dai Y, Fritzmann J, Birchmeier W. 2011. BCL9-2 promotes early stages of intestinal tumor progression. Gastroenterology 141: 1359-1370.e3.

Callaerts P, Halder G, Gehring WJ. 1997. PAX-6 in development and evolution. Annu Rev Neurosci 20: 483-532.

Cantù C, Valenta T, Hausmann G, Vilain N, Aguet M, Basler K. 2013. The Pygo2-H3K4me2/3 interaction is dispensable for mouse development and Wnt signaling-dependent transcription. Development 140: 2377-2386.

Deka J, Wiedemann N, Anderle P, Murphy-Seiler F, Bultinck J, Eyckerman S, Stehle J-C, André S, Vilain N, Zilian O, et al. 2010. Bcl9/Bcl91 are critical for Wnt-mediated regulation of stem cell traits in colon epithelium and adenocarcinomas. Cancer Res 70: 6619-6628.

de la Roche M, Rutherford TJ, Gupta D, Veprintsev DB, Saxty B, Freund SM, Bienz M. 2012. An intrinsically labile $\alpha$-helix abutting the BCL9binding site of $\beta$-catenin is required for its inhibition by carnosic acid. Nat Commun 3: 680.

Fiedler M, Sánchez-Barrena MJ, Nekrasov M, Mieszczanek J, Rybin V, Müller J, Evans P, Bienz M. 2008. Decoding of methylated histone H3 tail by the Pygo-BCL9 Wnt signaling complex. Mol Cell 30: 507-518.

Hill RE, Favor J, Hogan BL, Ton CC, Saunders GF, Hanson IM, Prosser J, Jordan T, Hastie ND, van Heyningen V. 1991. Mouse small eye results from mutations in a paired-like homeobox-containing gene. Nature 354: $522-525$.

Hogan BL, Horsburgh G, Cohen J, Hetherington CM, Fisher G, Lyon MF. 1986. Small eyes (Sey): a homozygous lethal mutation on chromosome 2 which affects the differentiation of both lens and nasal placodes in the mouse. J Embryol Exp Morphol 97: 95-110.

Kawamoto SA, Coleska A, Ran X, Yi H, Yang C-Y, Wang S. 2012. Design of triazole-stapled BCL9 $\alpha$-helical peptides to target the $\beta$-catenin/B-cell CLL/lymphoma 9 (BCL9) protein-protein interaction. J Med Chem 55: 1137-1146.

Kramps T, Peter O, Brunner E, Nellen D, Froesch B, Chatterjee S, Murone M, Züllig S, Basler K. 2002. Wnt/wingless signaling requires BCL9/ legless-mediated recruitment of pygopus to the nuclear $\beta$-cateninTCF complex. Cell 109: 47-60.

Lang RA. 2004. Pathways regulating lens induction in the mouse. Int $I$ Dev Biol 48: 783-791.

Li B, Mackay DR, Ma J, Dai X. 2004. Cloning and developmental expression of mouse pygopus 2, a putative Wnt signaling component. Genomics 84: 398-405.

Li B, Rhe C, Teng A, Bilanchone V, Munguia JE, Hu M, Jessen S, Piccolo S, Waterman ML, Dai X. 2007. Developmental phenotypes and reduced Wnt signaling in mice deficient for pygopus 2. Genesis 45: 318-325.

Machon O, Kreslova J, Ruzickova J, Vacik T, Klimova L, Fujimura N, Lachova J, Kozmik Z. 2010. Lens morphogenesis is dependent on Pax6-mediated inhibition of the canonical Wnt $/ \beta$-catenin signaling in the lens surface ectoderm. Genesis 48: 86-95.
Mani M, Carrasco DE, Zhang Y, Takada K, Gatt ME, Dutta-Simmons J, Ikeda H, Diaz-Griffero F, Pena-Cruz V, Bertagnolli M, et al. 2009. BCL9 promotes tumor progression by conferring enhanced proliferative, metastatic, and angiogenic properties to cancer cells. Cancer Res 69: $7577-7586$.

Mosimann C, Hausmann G, Basler K. 2009. $\beta$-Catenin hits chromatin: regulation of Wnt target gene activation. Nat Rev Mol Cell Biol 10: 276-286.

Nair M, Nagamori I, Sun P, Mishra DP, Rhéaume C, Li B, Sassone-Corsi P, Dai X. 2008. Nuclear regulator Pygo2 controls spermiogenesis and histone H3 acetylation. Dev Biol 320: 446-455.

Parker DS, Jemison J, Cadigan KM. 2002. Pygopus, a nuclear PHD-finger protein required for Wingless signaling in Drosophila. Development 129: $2565-2576$.

Schwab KR, Patterson LT, Hartman HA, Song N, Lang RA, Lin X, Potter SS. 2007. Pygol and Pygo2 roles in Wnt signaling in mammalian kidney development. BMC Biol 5: 15 .

Smith AN, Miller L-AD, Song N, Taketo MM, Lang RA. 2005. The duality of $\beta$-catenin function: a requirement in lens morphogenesis and signaling suppression of lens fate in periocular ectoderm. DeV Biol 285: 477-489.

Song N, Schwab KR, Patterson LT, Yamaguchi T, Lin X, Potter SS, Lang RA. 2007. pygopus 2 has a crucial, Wnt pathway-independent function in lens induction. Development 134: 1873-1885.

Städeli R, Basler K. 2005. Dissecting nuclear Wingless signalling: recruitment of the transcriptional co-activator Pygopus by a chain of adaptor proteins. Mech Dev 122: 1171-1182.

Takada K, Zhu D, Bird GH, Sukhdeo K, Zhao J-J, Mani M, Lemieux M, Carrasco DE, Ryan J, Horst D, et al. 2012. Targeted disruption of the BCL9/ $\beta$-catenin complex inhibits oncogenic Wnt signaling. Sci Transl Med 4: 148ra117.

Tang M, Yuan W, Fan X, Liu M, Bodmer R, Ocorr K, Wu X. 2013. Pygopus maintains heart function in aging Drosophila independently of canonical Wnt signaling. Circ Cardiovasc Genet 6: 472-480.

Tang M, Yuan W, Bodmer R, Wu X, Ocorr K. 2014. The role of pygopus in the differentiation of intracardiac valves in Drosophila. Genesis 52: $19-28$.

Thompson B, Townsley F, Rosin-Arbesfeld R, Musisi H, Bienz M. 2002. A new nuclear component of the Wnt signalling pathway. Nat Cell Biol 4: 367-373.

Valenta T, Gay M, Steiner S, Draganova K, Zemke M, Hoffmans R, Cinelli P, Aguet M, Sommer L, Basler K. 2011. Probing transcriptionspecific outputs of $\beta$-catenin in vivo. Genes Dev 25: 2631-2643.

Xie Q, Yang Y, Huang J, Ninkovic J, Walcher T, Wolf L, Vitenzon A, Zheng D, Götz M, Beebe DC, et al. 2013. Pax6 interactions with chromatin and identification of its novel direct target genes in lens and forebrain. PLOS ONE 8: e54507.

Yang Y, Cvekl A. 2005. Tissue-specific regulation of the mouse $\alpha$ Acrystallin gene in lens via recruitment of Pax6 and c-Maf to its promoter. J Mol Biol 351: 453-469. 


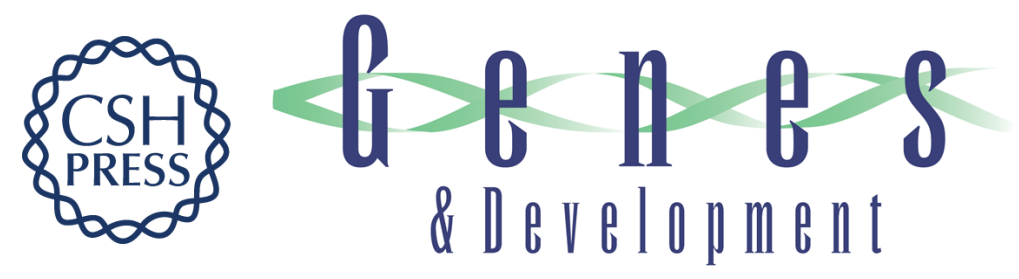

\section{Pax6-dependent, but $\beta$-catenin-independent, function of Bcl9 proteins in mouse lens development}

Claudio Cantù, Dario Zimmerli, George Hausmann, et al.

Genes Dev. 2014, 28:

Access the most recent version at doi:10.1101/gad.246140.114

\section{Supplemental http://genesdev.cshlp.org/content/suppl/2014/09/02/28.17.1879.DC1 Material}

References This article cites 33 articles, 11 of which can be accessed free at: http://genesdev.cshlp.org/content/28/17/1879.full.html\#ref-list-1

Creative This article is distributed exclusively by Cold Spring Harbor Laboratory Press for the first Commons six months after the full-issue publication date (see

License http://genesdev.cshlp.org/site/misc/terms.xhtml). After six months, it is available under a Creative Commons License (Attribution-NonCommercial 4.0 International), as described at http://creativecommons.org/licenses/by-nc/4.0/.

Email Alerting Receive free email alerts when new articles cite this article - sign up in the box at the top Service right corner of the article or click here.

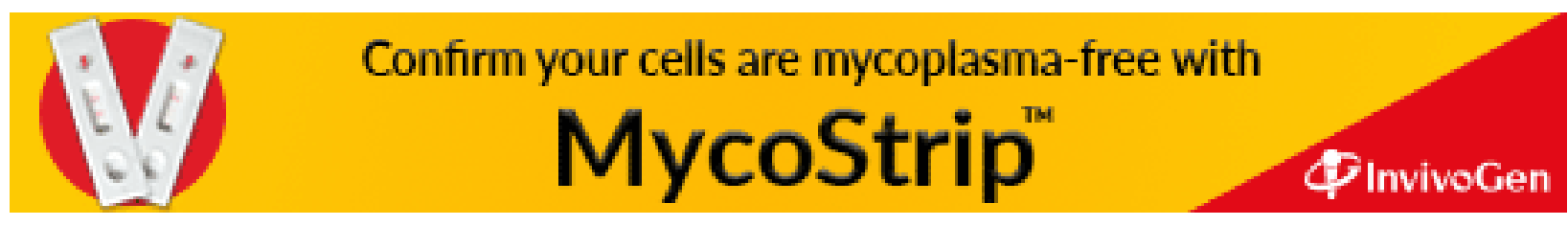

